\title{
In vivo antitrypanosomal activity of Garcinia hombroniana aqueous extract
}

\begin{abstract}
The anti-Trypanosoma evansi activity of Garcinia hombroniana (seashore mangosteen) leaves aqueous extract was tested on experimentally infected Sprague-Dawley rats. Treatment of infected rats with $\mathrm{G}$. hombroniana extract resulted in a significantly extended post-infection longevity $(p<0.05)$, compared to the untreated control group. The possible mode of antitrypanosomal effect of the plant extract was also investigated on cultured T. evansi in HMI9 medium with the addition of $25 \mu \mathrm{g} / \mathrm{ml} \mathrm{G}$. hombroniana aqueous extract. It was observed that the addition of $\mathrm{G}$. hombroniana extract resulted in the inhibition of trypanosomal kinetoplast division, with no significant inhibitory effect on nuclear division. It is concluded from the current study that the aqueous extract of G. hombroniana has a potential antitrypanosomal activity through the inhibition of kinetoplast division, as one of the possible mechanisms of its antitrypanosomal effect. This plant could serve as a possible source of new antitrypanosomal compounds.
\end{abstract}

Keyword: Garcinia hombroniana; Trypanosoma evansi; Trypanosomiasis; Surra 